\title{
The Principles of Design for Homemade Small Household Appliances*
}

\author{
Xinghe Wang \\ College of Electromechanical Engineering and Automation \\ Liming Vocational University \\ Quanzhou, China
}

\begin{abstract}
Targeting homemade small household appliances, this article analyzes the status quo of existing product designs and major problems on the basis of user's demands, product shape as well as enterprise cases, and raises three principles for the homemade small household appliances design namely safety, convenience and indication. Researches show that the safety is a foundation, the convenience is a direction and the indication is improvement.
\end{abstract}

Keywords-homemade small household appliances; design principle; product design

\section{INTRODUCTION}

Going with the continuous improvement of the people's living levels, small household appliances have been becoming more and more popular. Take the small household coffee machines as an example, roughly estimated, the consumption of small coffee machines in Chinese families is growing at a speed of $25 \%$ per year, increasing in a strong momentum, which are in a great need. Currently, the small household appliance suppliers at home are mainly copying the systems of foreign companies for which the suppliers act as OEMs, they are in the lack of a clear market positioning and poor in innovation. Designs and researches targeting homemade small household appliances are mainly focusing on two aspects: (1) Researches are conducted on the homemade household appliances from the industrial development and production design, such as Xiao Jun [1] through the analysis of status quo and existing technique of small household appliance industry, the author gets the development characteristics and trends of small household appliances in the future. Zhu Xiujuan [2] proposes that the contradiction matrix and innovation principle stated in TRIZ theory be used for analysis to improve the production and design flow of small household appliances so as to create a resolution for the production and design of small household appliances. (2) Industrial design procedures and methods can be used for the product design from humanization and individualization. Tan Ning[3] ,based on characteristics of small household appliances, points out that it is inevitable to integrate humanized factors to the homemade small household appliances. Yang Shouyan [4] discusses the possibility of feminine elements in small household appliances

*Fund project: Middle Age and Young Teachers Educational Scientific Research Program of Fujian Educational Department (JAT160835); Genera project of Liming Vocational University in 2017 (LW201704). design. In this article, targeting homemade small household appliances for the research, this author discusses a series of factors which may influence the small household appliances design and takes the corporate project practices into consideration to raise three principles for the design, aiming to increase the added value of products and make it be reference for the improvement and design of products of the same type.

\section{SAFETY PRINCIPLE}

According to the Maslow's psychological needs analysis, safety needs are a basic need followed by physiological needs that must be met, so the design of any small household appliances must be based on the safety, which consists of safety elements and safety design principle.

\section{A. Safety Factors}

The safety design of homemade small household appliances must follow the industrial safety standards. Currently there are two safety standard systems for global small household appliances around the world: IEC standard and UL standard, for China is a member of IECEE, so the IEC standard systems are adopted and two batches of product catalogs for compulsory safety certification have been promulgated in the country.

The design of homemade small household appliances must strictly abide by the domestic electronic product safety standards. Though the design requirements of various small household appliances differ, yet as far as the safety design, the elements concerned are almost the same, mainly including four points as follows: circuit design, structural design, material selection and safety mark. The circuit design is to reduce the local extreme temperature rise or fire due to over current in the circuit under normal conditions or in failure [5]; the structural design is to ensure the structure to own sufficient strength and stiffness, as well as appropriate anti-shock performance and body injuries protection. The material selection is to ensure the structure and strength of small household appliances, also having functions of insulation against electric shock and fire; the safety mark is an important means to ensure the operation and repair of small household appliances, refer to "Fig. 1" safety mark, which has functions of warning and indication, facilitating consumers to use correctly. 


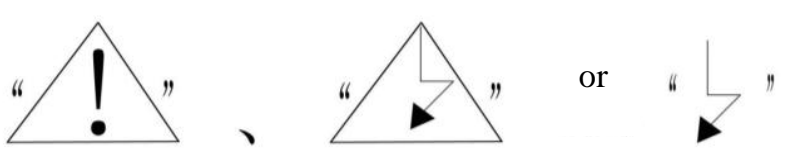

Fig. 1. Warning mark of domestic coffee machines.

\section{B. Safety Design}

The safety design of small household appliances must follow some rules, and its principles applicable can be summarized as electric shock and fire prevention, high temperature and mechanical injuries prevention as well as explosion and radiation prevention.

Electric shock and fire prevention: it means that insulating components under safety standards shall be selected correctly for the small household appliance design, and the accessible parts shall be structurally insulated from the dangerous voltage components on the product. Besides, it shall be protected from fire due to overheat and the fire spread, so the parts which produce heat easily shall be adjusted and limited on the circuit and structure, preventing from fire sources, flame-resistant materials shall be adopted for the parts which are easy to catch fire and spread. "Fig. 2" Drip circuit design, of which, the heat conducting plate height is $40 \mathrm{~mm}$ at least, and the water tank height is over $80 \mathrm{~mm}$.

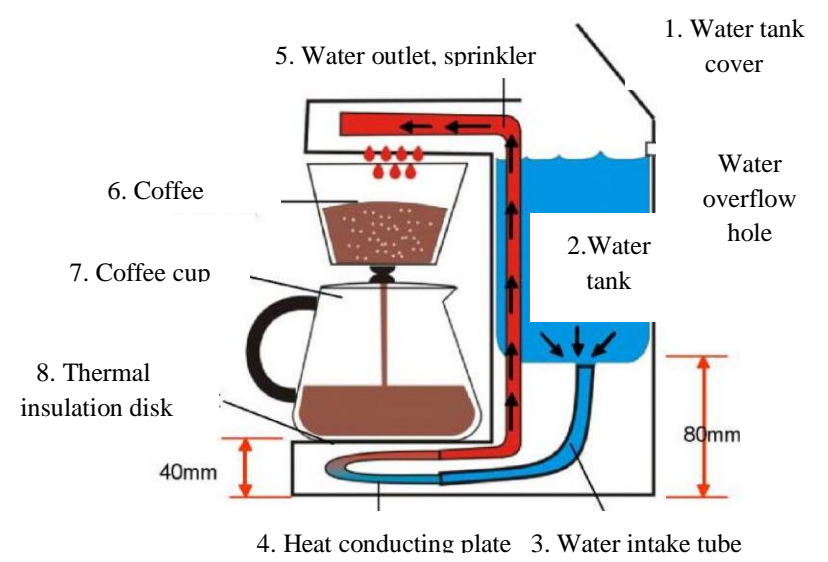

Fig. 2. Drip circuit design diagram.

High temperature and mechanical injuries prevention: it means that when designing small household appliances, it shall prevent over high temperature of local parts from injuring the user or from the electric shock by the softened materials due to over high temperature, therefore the parts which are easy to produce over current or heat on the circuit shall be limited so as to protect the products from danger due to over current or over heat under normal or abnormal conditions; besides, during the use of small household appliances, it needs to prevent local parts of the product from injuring the users or the inclination of unstable supports or moving parts from injuring the users, besides, it shall avoid sharp edges or burrs.

Explosion and radiation prevention: it means that when designing small household appliances, it needs to prevent the small household appliances from body injuries due to explosion under large pressure while using. And more attentions shall be paid to the parts with huge pressure thereon, such as espresso high pressure boiler, namely the radiation restrictions shall be below the given value when the small household appliances are used, and the materials with less radiation shall be used as possible as we can.

\section{CONVENIENCE}

The convenience design of small household appliances means that the product use should meet objective laws, conform to consumers' physical and psychological needs, making it rapid and convenient to use, and it is a basic requirement for the "human-oriented" design concept.

\section{A. Factors for Convenience}

When designing the convenience for small household appliances, we should not only fully consider user's experience, professional and technical background, but also know user's age and gender differences and so on, which consist of three points namely ergonomics, product linguistics and design psychology.

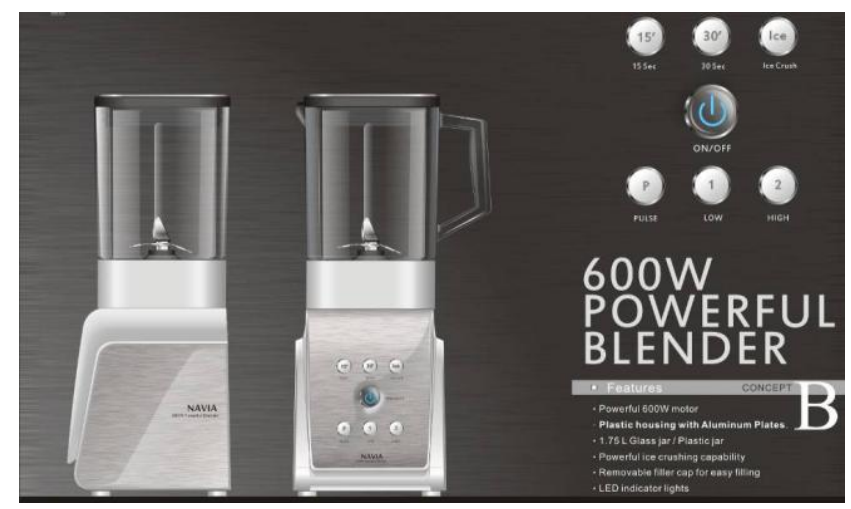

Fig. 3. Buttons of small household appliances.

Ergonomics: it consists of three aspects: human scale, environmental factors and information feedback. (1) Human scale: human scale will vary with region, gender, age groups and so on, of which, the regional differences are large, which shall not be copied directly. For example, the coffee capsule capacity at home mostly copy foreign standards, but failing to consider that Chinese consumers have low demands for caffeine, and the product fails to be developed according to the actual situations at home; (2) Environmental scale: it consists of visual environment, sound sensation environment and so on. Take "Fig. 3" buttons of small household appliances as an example, it is a most basic application of a point in the shape structure, generally, it is expressed by higher brightness of color, showing locations and apparent sights, which is also supported by prompt sound, which is an important factor to be well considered in the design of small household appliances; (3) Information feedback: it means information acquired by vision and other perceptions as well as the interaction between information and control. Logical information and feedback may increase the convenience to users while reducing errors; Product linguistics: during the design of convenience, product 
linguistics emphasizes the product's recognition, logical manmachine interface as well as simple and understandable operation procedures. Refer to "Fig. 4" the milk froth machine adopts one-touch operation, easy to identify and operate and equipped with a reasonable man-machine interface, the user may heat while making milk froth, when the temperature reaches $70^{\circ} \mathrm{C}$, it will cut off the power supply automatically so as to maximize the product value.

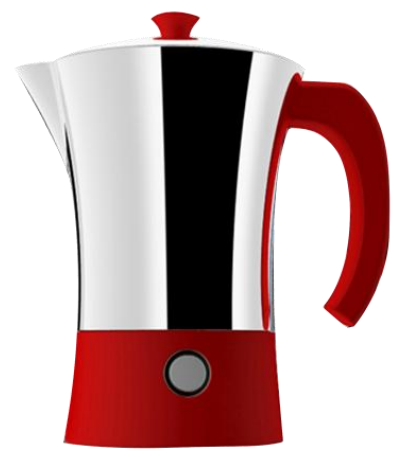

Fig. 4. Milk froth machine design.

Design psychology: according to the subjects, it consists of designer's psychology, consumer's psychology and user's psychology. The convenience design of small household appliances shall give more consideration to the user's psychology, when designing, the designers must fully study the user's psychology, consider factors such as vision, auditory sense, tactile sense and body size to master possible problems for improvement so as to meet the user's physical and psychological requirements on the product convenience.

\section{B. Convenience Design}

The convenience of small household appliances shall mainly be focused on five points namely preset function, concept model, visibility and rational use restriction, information matching and feedback.

The preset function means that a product is considered to have some performances and its actual performance, primarily referring to the basic performances which determine how to the product is used [6]. During the convenience of small household appliances, sound preset function may greatly guide the user to operate in order to achieve more convenient and efficient operation;

Concept model: it refers to general psychological modes and models [7] which are formed by the people via training and experience on environment, self and what they contact. During the convenience design of small household appliances, a good concept model may predict the results of operational behaviors so as to reduce the user's time to learn, while providing executable clues and basis to solve the product troubles.

Visibility and rational use restriction: Through shapes and colors, the designer shows user the product functions and correct operation methods, making it easy for user. During the convenience design of small household appliances, a logical visual design may give the user correct indication, it shows the hidden parts of the product, greatly improving the product convenience; the well designed display device may avoid unnecessary complex operation; the well designed operation prompt sound may increase the visibility of the product. In the meantime, the designer should take possible errors into consideration, using various restrictions to remind and force the user to avoid the improper operation. Refer to "Fig. 5" the kneading machine design, the design of product handle is simplified visually to make it easy for operation, which greatly improve the comfort of the product.

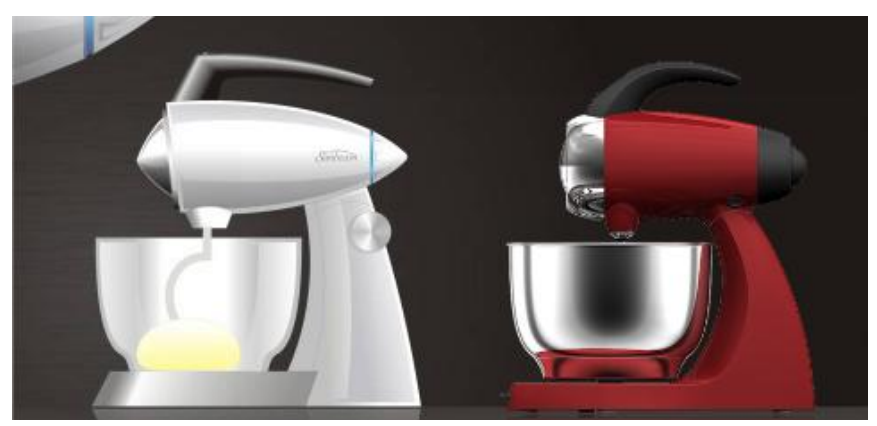

Fig. 5. Dough kneading machine design.

Information matching and feedback principle: it means that the designer compares the users' thinking patterns to make the product use meet what the users expect, making the feedback match with the users' intention, which are shown through correct ways. During the convenience design of small household appliances, a good matching is critical, which can save the user's time to learn, reduce the improper operation and achieve the convenience, besides, the design should consider that the user's operation can get timely and obvious feedback information, which will remind the user via images and symbols stored outside to reduce its memory burden. During the convenience design, the timely information feedback enhances the operator's perception and skills, making the complicated operation simple and convenient.

\section{STRESSING INDICATION}

The indication of small household appliances refers to a design method which describe correctly and logically the product's function and features, which is used to serve the small household appliances (material and spiritual functions), having an important significance in instruction.

\section{A. Factorof Indication}

The indicative design of small household appliances shall not only fully serve functions but also correctly distinguish the decoration from indication, avoiding unnecessary visual misleading. Its main contents include the product form and selection of operation and use, which are influenced by restricted by factors of their own and operating environment.

Product form: The form plays a leading role in the indicative design of small household appliances, which is an illation of its function, feeling and value. For example, the lines with different characteristics may bring users different feeling, the straight line shows intensity, sharpness and logic; the curve shows elegance, please and changes, and the smart 
changes in the line form, length and thickness or other proportion may help use design diverse and colorful structural forms[8]. Different forms show different emotions, for example, square, rectangle and round show a beauty of simplicity, clearness and order; triangle or irregular forms bring a feeling of activity and relax. Refer to "Fig. 6" surfaces applied on small household appliances, the rectangular metal panel highlight the product's stability and order, bringing the user a sense of safety on the product.

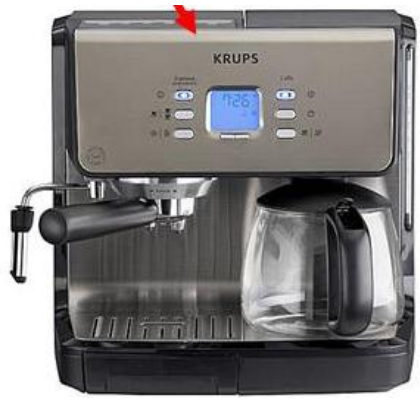

Fig. 6. Surfaces applied on small household appliances.

Selection of operation mode: generally the operation of small household appliances is based on meeting the ergonomic requirements, the operator's habits and psychology, fully attracting the user's attention and interest in order to make the product user more secure, comfortable and logical. During the indicative design of small household appliances, we should fully consider the product's operation mode and order, after continuous operation or operation ends, there will be corresponding prompt and feedback, besides, the operational tasks are simplified.

\section{B. Indicative Design}

The indicative design of small household appliances mainly consists of indication of the form of the product in use and indication for the production operation.

Indication of product form in use: the form should be implied via similar shape, as shown in "Fig. 7", design of handle with horizontal texture of the coffee machine, it shall not only make it comfortable for the manual operation but also show its mode to grasp. The form can hint the operation way via causal association of styling, for example, make use of the thick or thin textures and numbers around the knob of the coffee machine to show the precision of the knob adjustment. The final shape can make use of the surface texture and color to hint the operation method to attract the attentions.

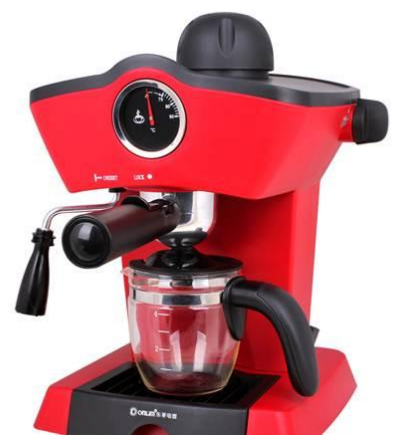

Fig. 7. Design of handle with horizontal texture of the coffee machine.

Indication of product operation: During the convenience design of small household appliances, we must make sure of the consumers concerned, consider their consumption habits, master the basic, necessary and logical needs of the consumer groups, deeply grasp the consumer's understanding of the product, and ensure the operation methods are simple and easy to learn. Main ways include: make use of new materials and new technique to reorganize the operational tasks and improve the feedback mechanism so as to display the information needed in a more simple way, enhance the perception, in the meantime, automation techniques can be adopted to simplify the operational tasks and reduce the operational burden. Finally, the operation mode shall be limited logically to avoid the improper operation or mistakes in operation, and for the operation which is unable to reverse, compelling function design can be adopted.

\section{CONCLUSION}

Small household appliances are closely related to the daily life of the people, whose design is not a simple functional superposition; it needs to meet the needs of consumers continuously going with the market development. During the development, it must follow some design principles, such as safety, sharing, simplicity and convenience. After analyzing the components and design principles, this article points out that the safety is a foundation, the convenience is a direction, and the indication is an improvement, aiming to provide theoretical basis for the design and research of homemade small household appliances, making the design targeted and go as expected.

\section{REFERENCES}

[1] Xiao Jun, Characteristics and Trend of Small Household Appliances in the Future [J] Electronic Technology, 2007（8-9）: 146.

[2] Zhu Xiujuan, TRIZ Theory-based Innovative Design of Small Household Appliances [J] Manufacturing Automation, 2015 (10) :134135 .

[3] Tan Ning, Humanistic Design of Small Household Appliances [J] Journal of Southern Yangtze University (Humanities \& Social Sciences Edition), 2013 (3) :104-105.

[4] Yang Shouyan, Application and Embodiment of Feminine Design on Small Household Appliances [J] Art Science \& Technology, 2014(1) : 29-30

[5] Wu Li'an, Zhang Sha, Introduction to Safety Design of Electronic Products, Shenzhen Electronic Products Quality Test Center

[6] Yang Ling, Zhang Mingchun, Design Concept and Method for Sharing of Public Facilities [J], China Science and Technology Information, 2008 (23) : 161-162.

[7] Chen Hanqing, Product Design [M]. Wuhan: Huazhong University of Science \& Technology Press, 2005. 25-30

[8] Li Gongwei, On Graphic Tension in the Graphic Design [J]. Northeast Normal University, Thesis for Master's Degree, 2005: 108 